\title{
Sirt1 regulates testosterone biosynthesis in Leydig cells via modulating autophagy
}

\section{Dear Editor,}

Steroid hormones are crucial signal molecules that regulate a large number of physiological and developmental processes. Testosterone is the key steroid hormone required for the development of male characteristics and also supports the physiology of the male reproductive system (Sinclair et al., 2015). Testosterone is primarily produced by the Leydig cells residing in the testicular interstitium. The cholesterol acts as a substrate for the biosynthesis of testosterone. Since steroidogenic cells are capable of storing only very little hormone, rapid synthesis of hormone requires the mobilization of the precursor cholesterol, chiefly stored as intracellular lipid droplets (LDs) (Danielsen et al., 2016). Leydig cells are the major sites to produce testosterone, there are extremely active autophagy in them, and a decline in steroidogenesis has also been associated with the decline of autophagic flow. Moreover, the disruption of autophagy leads to decreased intracellular LDs, and therefore affects testosterone synthesis in the Leydig cells (Danielsen et al., 2016; Gao et al., 2018).

Sirtuin 1 (SIRT1) is one of the members of NAD-dependent protein deacetylase which regulates multiple cellular functions such as metabolism, apoptosis, and autophagy (Cohen et al., 2004; Chang et al., 2015). SIRT1 is necessary for fertility in mice, it participates in the differentiation of spermatogenic stem cells, acrosome biogenesis, and histone-to-protamine transition during spermatogenesis (Liu et al., 2017). However, the functional role and underlying mechanism of SIRT1 in testosterone biosynthesis are yet unknown.

To explore the role of Sirt1 in testosterone biosynthesis, Sirt ${ }^{F I F}$ mice were crossed with SF1-Cre mice to generate steroidogenic cell-specific Sirt1-knockout mice, and the knockout efficiency of SF1-Sirt1 $1^{-1-}$ mice was further confirmed by Western blot (Fig. 2E). We found no difference in testis morphology (Fig. S1A) and the sperm count in cauda epididymis (Fig. S1B) of Sirt1 ${ }^{F / F}$ and SF1-Sirt1 $1^{-/-}$mice. However, the mating efficiency and pregnancy rate of SF1$\mathrm{Sirt}^{-1-}$ mice were significantly decreased compared to Sirt ${ }^{F / F}$ mice (Fig. S2). To investigate reduced mating efficiency and docile behavior, we carried out sexual behavior analysis. SF1-Sirt $1^{-1-}$ mice showed a significantly longer latency (Fig. 1A) and mounted targeted females less frequently and with a shorter mounting duration (Fig. 1B and 1C) compared to the Sirt $1^{F / F}$ mice. However, sniffing frequency and duration was not significantly affected in SF1Sirt $1^{-1-}$ mice compared to control mice (Fig. 1D and 1E). In vertebrates, male sexual behavior is regulated by testosterone, thus we measured the serum testosterone concentration and found reduced testosterone levels in the sera of SF1-Sirt $1^{-1-}$ mice (Fig. 1F). Testosterone levels were also significantly reduced in the Leydig cells of SF1-Sirt ${ }^{-1-}$ mice (Fig. 1G). Together, these findings suggest that Sirt1-disruption results in a sharp decrease in testosterone and influence the sexual behavior of male mice, which is very similar to the symptoms of late-onset hypogonadism ( $\mathrm{LOH}$ ) (Swee and GAN, 2019).

Different factors affect serum testosterone levels (Chen et al., 2009; Midzak et al., 2009). We firstly measured luteinizing hormone (LH) and follicle-stimulating hormone (FSH) concentrations in the sera of both Sirt1 ${ }^{F / F}$ and SF1Sirt $1^{-1-}$ mice but found no significant differences in $\mathrm{LH}$ and FSH concentrations (Fig. S3A and S3B). Next, we focused on testosterone synthesis related factors. A large number of steroidogenic factors are involved in the transformation of cholesterol to testosterone, including $\mathrm{LH}$ receptor (LHCGR), lipoprotein receptors, cholesterol transporter STAR, and some steroidogenic enzymes CYP17A1, HSD3B1, CYP17A1 and HSD17B3 (Fig. S4A). Therefore, we measured the mRNA levels of these steroidogenic factors step by step from the initial raw material, cholesterol to testosterone, and found a significant decrease in these factors (Fig. S4). As HSD3B1 is an important enzyme involved in testosterone biosynthesis, we measured the mRNA and protein level of this enzyme and found a significant decrease in the levels of both mRNA and protein in the Leydig cells of SF1-Sirt $1^{-1-}$ mice (Fig. $1 \mathrm{H}-\mathrm{J}$ ). Next, we assessed the levels of HSD3B1 in testicular sections by immunofluorescence and noted a significant decrease in the Leydig cells of SF1Sirt $1^{-1-}$ mice as compared to those of Sirt $1^{F / F}$ mice (Fig. 1K). To further confirm these findings, we measured HSD3B1 activity and found a considerable decline in the Leydig cells isolated from SF1-Sirt1 ${ }^{-1-}$ mice compared to control mice (Fig. 1L). Thus, these findings suggest perturbed steroidogenesis in SF1-Sirt $1^{-/-}$mice. 


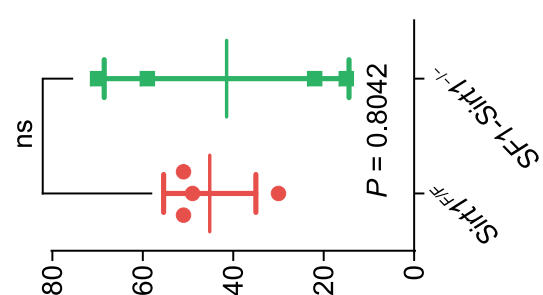

(s) әu!̣ bu!ı!!us

ш

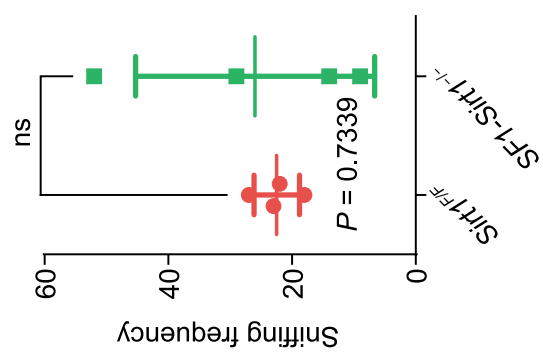

$\square$

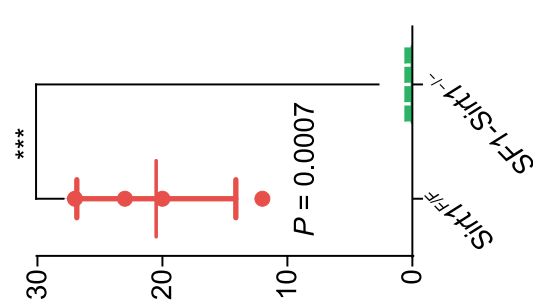

(s) uo!̣eınp bu!̣unow

。

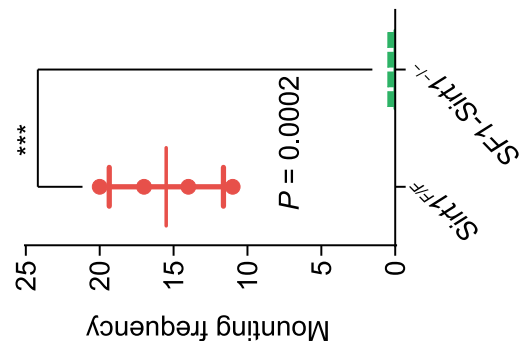

$\infty$

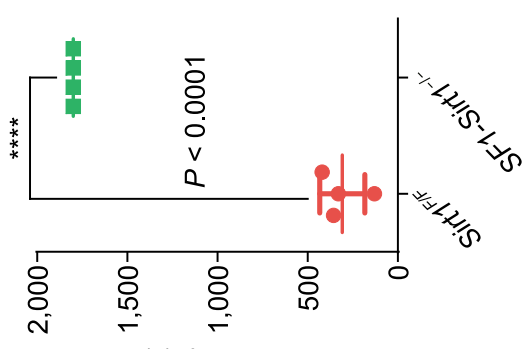

(s) Кэuәрา

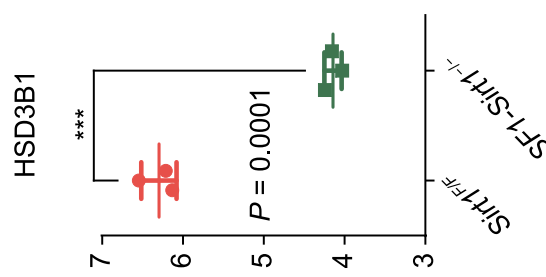

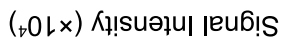

$\supset$
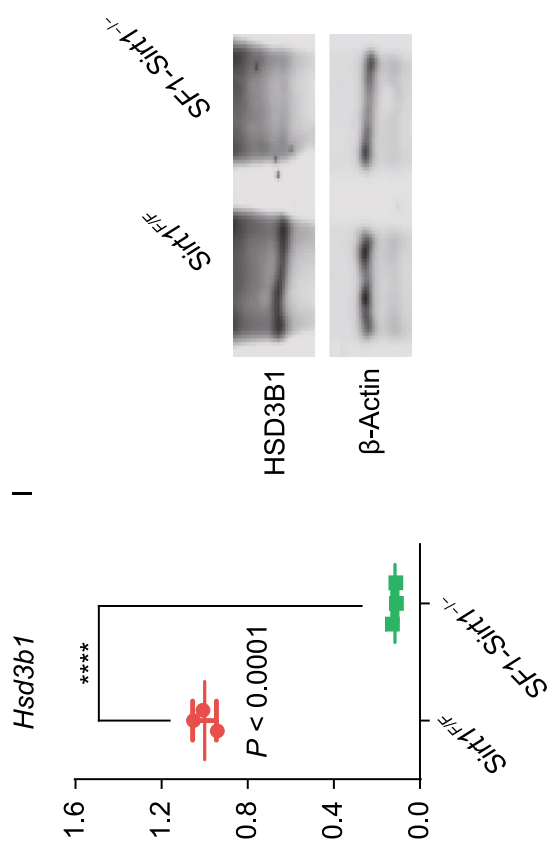

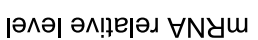

I

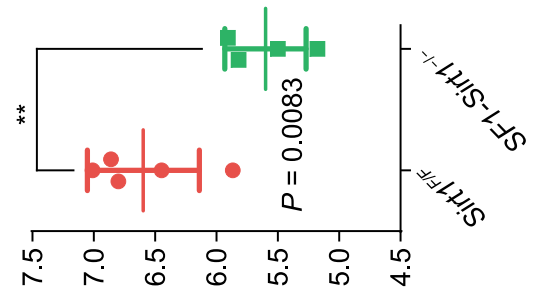

(ךu/6u) s||ə弓 6!рК夫ә

0

u! әuoגəłsolsə।

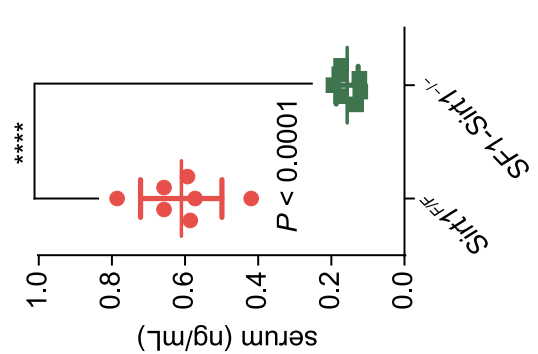

ᄂ

u! әuodə다이리
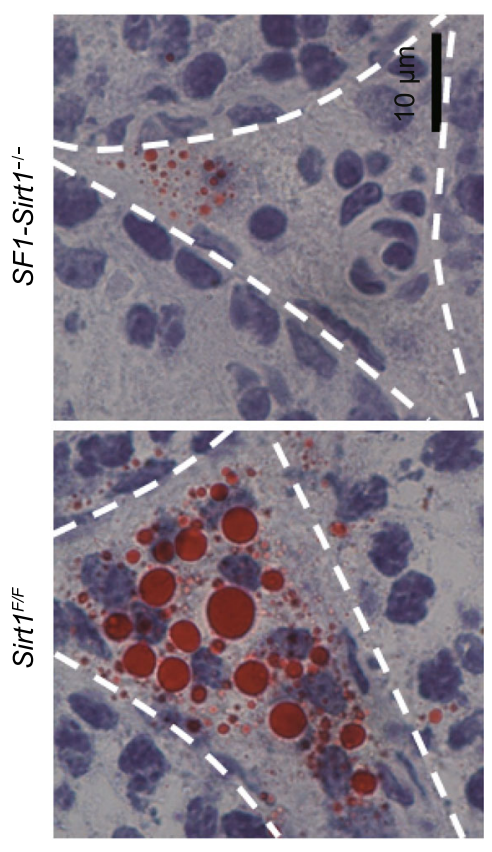

$z$

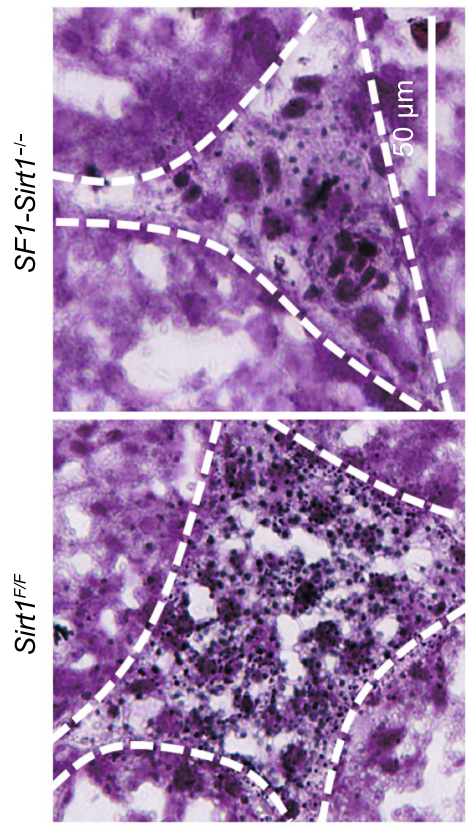


4 Figure 1. Sirt1 disruption in Leydig cells affects sexual behavior and interrupts steroidogenic activity. (A) The sexual behavior of SF1-Sirt $1^{-1-}$ mice was severely compromised as these mice showed prolonged latencies in mounting target females compared to that of Sirt1 ${ }^{F / F}$ mice. (B and C) Similarly, SF1-Sirt1 ${ }^{-1-}$ mice mounted targeted females less frequently and also showed a significantly decreased mounting duration compared to Sirt1 ${ }^{F / F}$ mice. (D and E) Sniffing frequency and sniffing time showed no obvious differences among Sirt $1^{F / F}$ and SF1-Sirt $1^{-1-}$ mice. (F) Testosterone levels in the sera of SF1-Sirt1 ${ }^{-1-}$ mice showed a significant reduction compared to Sirt ${ }^{F / F}$ mice. (G) Testosterone levels in the Leydig cells isolated from SF1-Sirt $1^{-1-}$ mice were decreased significantly compared to the Leydig cells isolated from Sirt ${ }^{F / F}$ mice. $(\mathrm{H})$ The relative mRNA level of $H s d 3 b 1$ was significantly decreased in the Leydig cells upon Sirt1 disruption. (I) Immunoblotting analysis of HSD3B1 showed a significant reduction in Sirt1-deficient Leydig cells compared to those obtained from Sirt1 ${ }^{F / F}$ mice. $\beta$-Actin was used as the loading control. (J) Quantification of the relative signal intensity of HSD3B1 shown in (I). Data being represented as mean \pm SD and ${ }^{* * \star *} P<0.0001$. (K) Immunofluorescence staining showed a significant reduction in the signal of HSD3B1 in the Leydig cells from SF1-Sirt1 ${ }^{-1-}$ mice compared to those of Sirt $1^{F / F}$ mice. (L) The activity of the steroidogenic enzyme HSD3B1 was significantly reduced in the Leydig cells from SF1-Sirt $1^{-1-}$ mice compared to those of Sirt $1^{F / F}$ mice. (M) An extreme reduction in lipid content was found in the Leydig cells of SF1-Sirt1 $1^{-1-}$ mice. BODIPY staining showed a sharp decrease in the number of LDs in the Leydig cells of Sirt1-deficient mice. (N) ORO staining performed on the testicular sections of SF1-Sirt $1^{-1-}$ mice showed a significant reduction of LDs in the Leydig cells compared to Sirt1 ${ }^{F / F}$ mice. (O) TC levels were significantly reduced in the Leydig cells of SF1-Sirt $1^{-1-}$ mice compared to the Leydig cells of Sirt1 ${ }^{F / F}$ mice. $(P)$ No obvious differences were found in the TC levels in the whole testes extracts of Sirt1 ${ }^{F / F}$ and SF1-Sirt $1^{-1-}$ mice. (Q) TG levels were significantly reduced in the Leydig cells of SF1-Sirt1 ${ }^{-1-}$ mice compared to the Leydig cells of Sirt ${ }^{F / F}$ mice. (R) No obvious difference was found in the TG levels in the whole testes extracts of Sirt $1^{F / F}$ and SF1-Sirt1 ${ }^{-1-}$ mice.

Cholesterol is utilized as major raw material to synthesize testosterone in the Leydig cells (Hu et al., 2010), therefore we carried out immunofluorescence analysis of BODIPY to detect the presence of cytoplasmic LDs, where cholesterol is stored as cholesterol esters (Ouimet et al., 2019). To our surprise, a significant decrease in LDs was observed in the testicular sections of SF1-Sirt1 $1^{-1-}$ mice (Fig. 1M). Similar results were also obtained using Oil Red $O(O R O)$ staining, where we found a clear decrease in LDs in SF1-Sirt $1^{-1-}$ mice (Fig. 1N). We further assessed total cholesterol (TC) levels and found a significant decrease in Leydig cells isolated from SF1-Sirt $1^{-1-}$ mice (Fig. 10) but not in whole testis extracts or sera (Figs. 1P and S5A). Triglycerides (TG) are also stored in LDs, so we next measured TG concentrations in Leydig cells. Similar to TC, TG levels were also reduced significantly in the Leydig cells and sera (Figs. $1 \mathrm{Q}$ and S5B), but not whole testis extracts (Fig. 1R) isolated from SF1-Sirt1 $1^{-1-}$ mice. Collectively, these results suggest that the testosterone deficiency in SF1-Sirt1 $1^{-1-}$ mice might come from inadequate cholesterol transport into the Leydig cells.

Thus, decrease of LDs and cholesterol in the Leydig cells of SF1-Sirt ${ }^{-1-}$ mice might be a result of decreased supplies of cholesterol. High-density lipoprotein (HDL) serves as the chief cholesterol source in testosterone biosynthesis in the Leydig cells. Therefore, we performed an in vitro cholesteroluptake experiment employing fluorescence-labeled cholesterol-rich lipoprotein 1,1'-dioctadecyl-3,3,3',3'-tetramethylindocarbocyanine perchlorate (Dil)-HDL in Leydig cells isolated from Sirt1 $1^{F / F}$ and SF1-Sirt1 $1^{-/-}$mice to find out whether SIRT1 participates in cholesterol uptake from HDL. A significant decrease in cholesterol absorption was observed in the Leydig cells of SF1-Sirt $1^{-1-}$ mice compared to Sirt1 ${ }^{F / F}$ mice (Fig. 2A). Together, our findings suggest that SIRT1 actively participates in cholesterol uptake for steroidogenesis in Leydig cells.

SR-BI has been described as a major lipoprotein receptor specifically involved in the uptake of HDL, LDL, or VLDL. Therefore, to explore whether SR-BI participates in cholesterol absorption, we measured SR-BI levels via immunoblotting and found a significant decrease in SR-BI levels in the Leydig cells isolated from SF1-Sirt $1^{-1-}$ mice compared to control mice (Fig. $2 \mathrm{~B}$ and $2 \mathrm{C}$ ). To further confirm these findings, we carried out immunofluorescence analysis of $\mathrm{SR}-\mathrm{BI}$ in testicular sections. A significant reduction in SR-BI signal was found in SF1-Sirt $1^{-/-}$testes compared to control testes (Fig. 2D). Together, reduced cholesterol in the Leydig cells of SF1-Sirt1 ${ }^{-1-}$ mice might represent perturbed cholesterol uptake as a result of SR-BI down-regulation.

SIRT1 has been reported to be an important regulator of autophagy by directly deacetylating LC3 and ATG7, thus affects their translocation from the nucleus to the cytoplasm (Chang et al., 2015). Therefore, we investigated the effects of Sirt1-knock out on autophagy flux and found that SQSTM1/p62 and LC3I (cytosolic form), but not LC3II (membrane-bounded form), accumulated in Leydig cells (Fig. 2E-H), suggesting that autophagic flux is indeed disrupted in the Leydig cells of SF1-Sirt1 ${ }^{-/-}$mice. To test the nucleocytoplasmic redistribution of LC3, we carried out immunofluorescence analysis of testicular sections from Sirt ${ }^{F / F}$ and SF1-Sirt $1^{-1-}$ mice and found a clear accumulation of LC3 in the nuclei of Sirt1-deficient Leydig cells (Fig. 2I). To further verify our immunofluorescence results, we examined the LC3 acetylation level in the Leydig cells isolated from Sirt $1^{F / F}$ and SF1-Sirt $1^{-/-}$mice and found a significant increase in LC3 acetylation in the Leydig cells of Sirt1-deficient mice (Fig. 2J and 2K). Thus, SIRT1 might regulate the autophagic process by modulating LC3 acetylation. Decrease in LAMP2 (marker of lysosomes or 


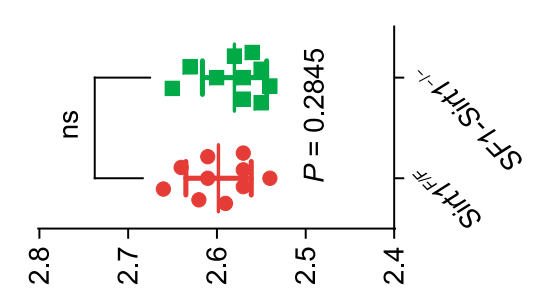

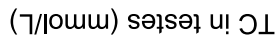

0

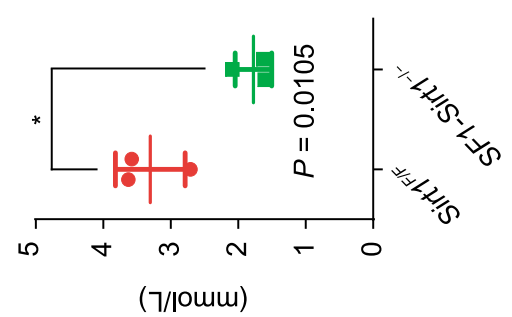

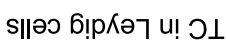

0
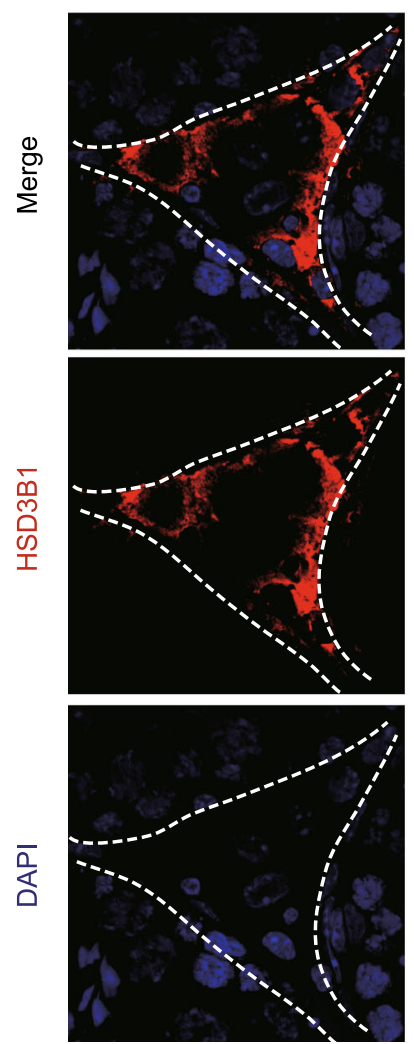

צ

--เน!S-เコS
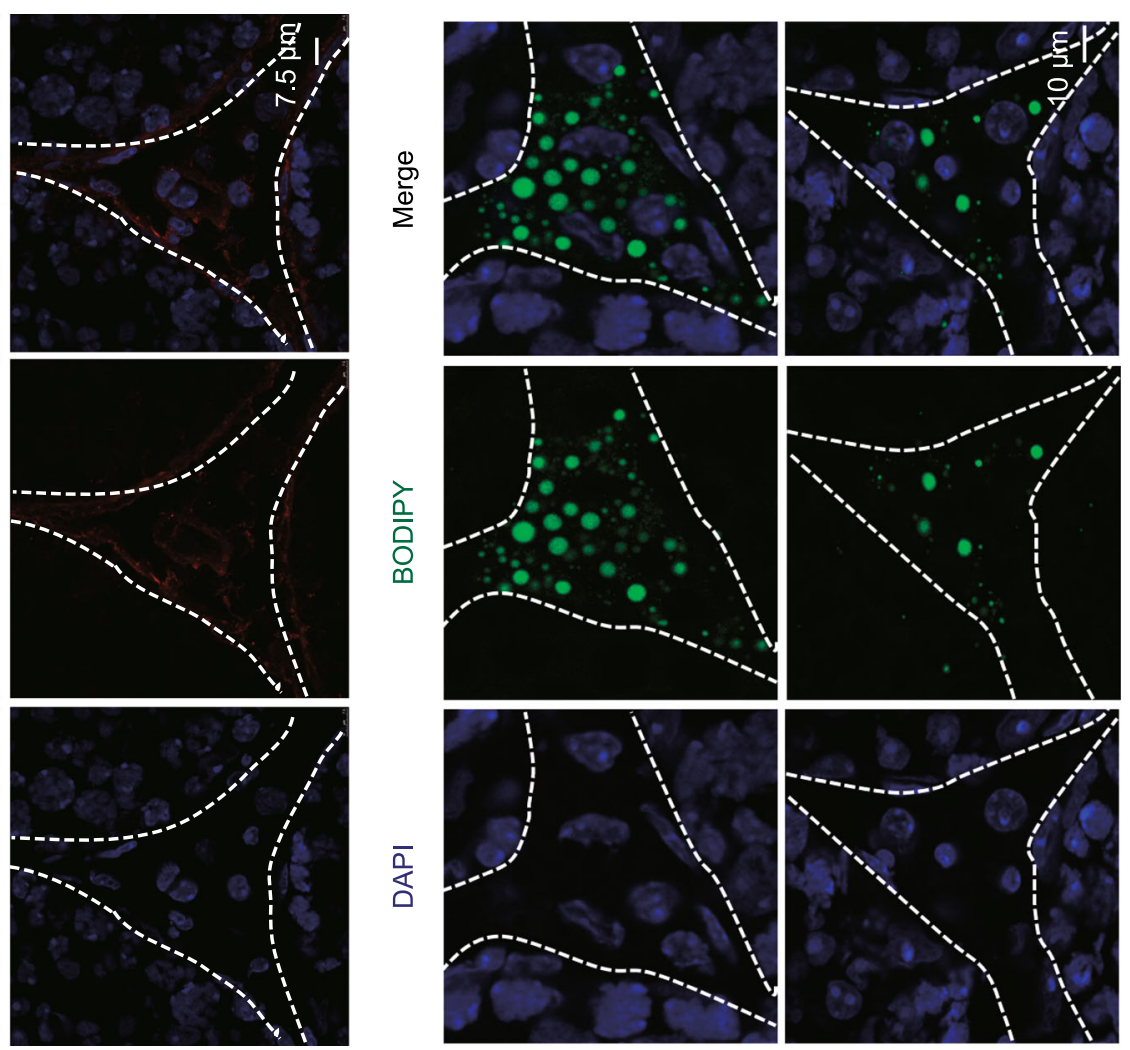

$\Sigma$

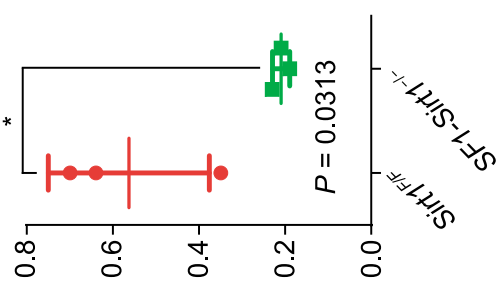

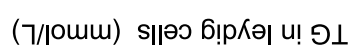
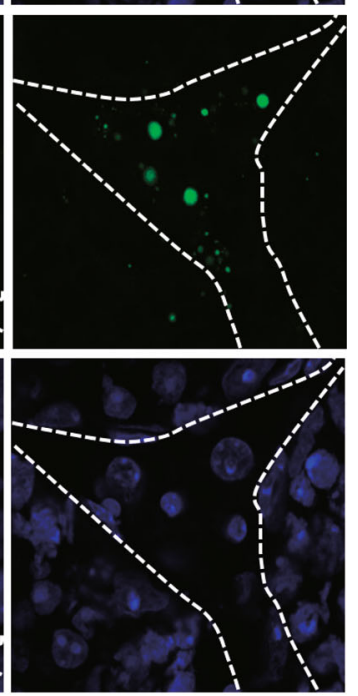

킨‼S

-ะ-เน!!S-เコS 


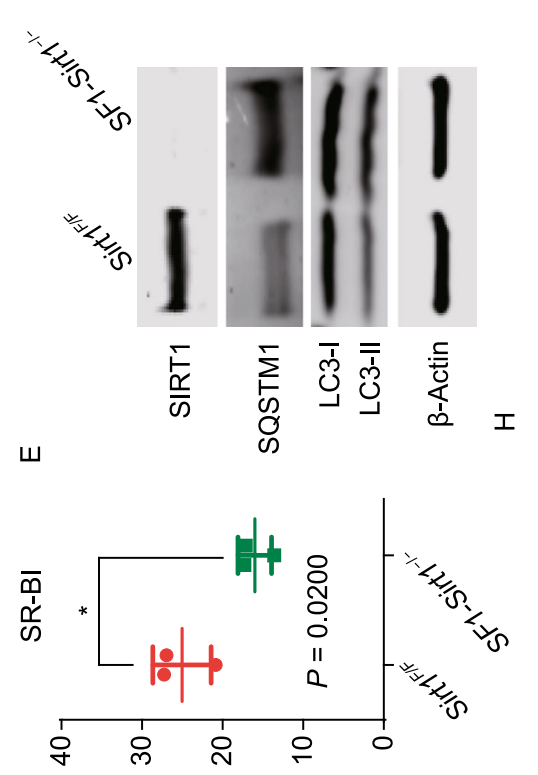

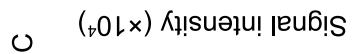
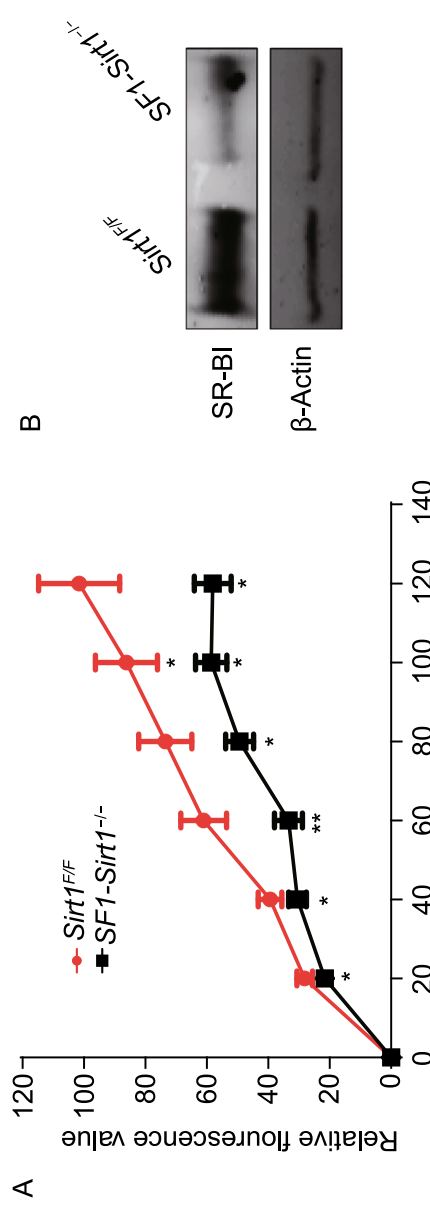

$\varangle$

ᄂ

4
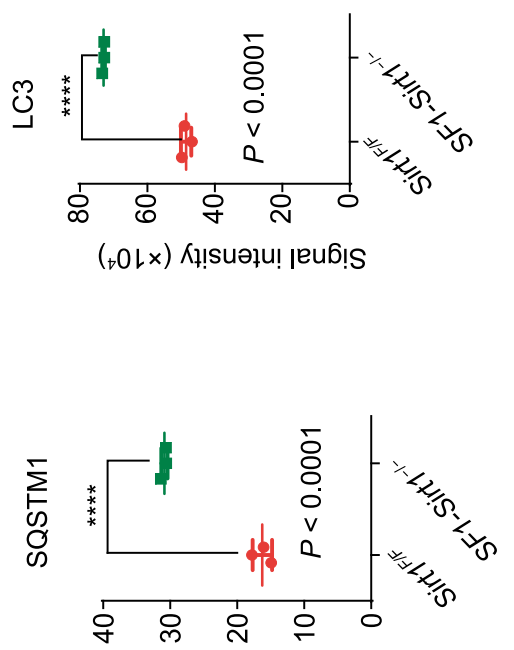

$\left.{ }_{(t} \mathrm{OLX}\right)$ Kł!suə]u! ןeu6!s
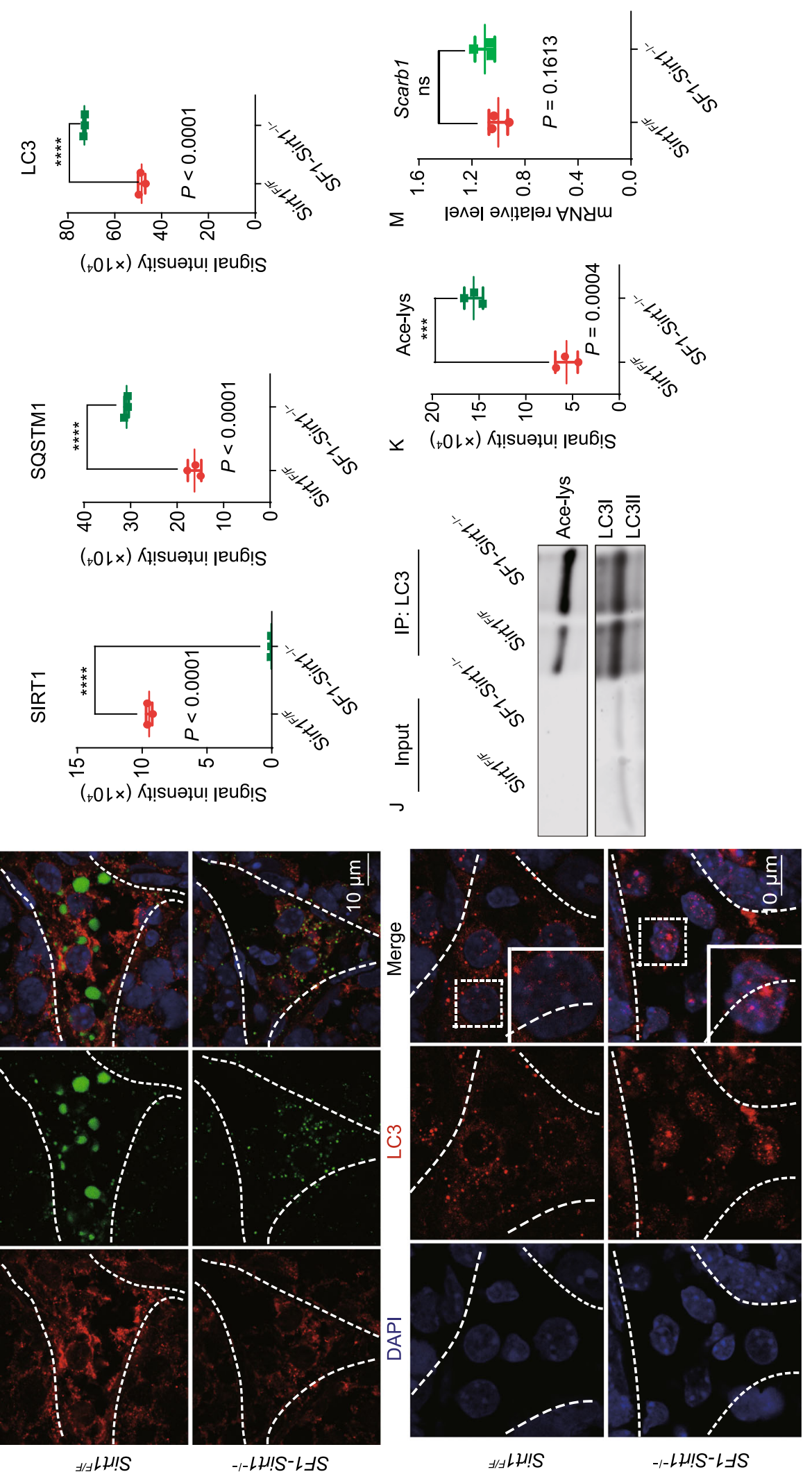
Figure 2. Sirt1 disruption impairs autophagy and hinders cholesterol uptake in Leydig cells. (A) Cholesterol (Dil-HDL) absorption was significantly reduced in the Sirt1-deficient Leydig cells compared to the Leydig cells of Sirt ${ }^{F / F}$ mice. (B) Immunoblotting analysis of SR-BI showed a significant reduction of SR-BI in Sirt1-deficient Leydig cells compared to Leydig cells of Sirt1 ${ }^{F / F}$ mice. $\beta$-Actin was used as the loading control. (C) Quantification of the signal intensity of SR-BI shown in (B). Data is represented as mean $\pm \mathrm{SD}$ and ${ }^{*} P<0.0001$. (D) Immunofluorescence staining of SR-BI showed a significant decrease in the Leydig cells of SF1-Sirt1 ${ }^{-1-}$ mice compared to the Leydig cells of Sirt ${ }^{F / F}$ mice. BODIPY staining showed a sharp decrease in the number of LDs in the Leydig cells of Sirt1-deficient mice compared to Sirt1 ${ }^{F / F}$ mice. (E) SIRT1 levels were significantly reduced in autophagydeficient Leydig cells compared with Sirt1 ${ }^{F / F}$ Leydig cells. SQSTM1/p62 and LC3 showed considerable accumulation in the Leydig cells of SF1-Sirt1 ${ }^{-1-}$ mice. (F) Quantification of the relative signal intensities of SIRT1 shown in (E). Data are represented as mean \pm SD and ${ }^{* * * *} P<0.0001$. (G) Quantification of the signal intensities of SQSTM1/p62 shown in (E). Data are represented as mean \pm SD and ${ }^{* * *} P<0.0001$. (H) Quantification of the signal intensities of LC3 shown in (E). Data are represented as mean \pm SD and ${ }^{* * * *} P<$ 0.0001 . (I) Immunofluorescence staining of LC3 showed a significant accumulation of LC3 puncta in the nuclei of Leydig cells of SF1Sirt $1^{-1-}$ mice. (J) Immunoprecipitation analysis to detect the acetylation level of endogenous LC3 in the Leydig cells of Sirt $1^{F / F}$ and SF1-Sirt1 ${ }^{-1-}$ mice. Western blot was used to analyze the immunocomplexes. (K) Quantification of the signal intensity of Ace-lys shown in $(\mathrm{J})$. Data are represented as mean $\pm \mathrm{SD}$ and ${ }^{* \star *} P<0.001$. (L) Immunofluorescence staining of LAMP2 showed a significant decrease in the Leydig cells of SF1-Sirt $1^{-1-}$ mice compared to the Leydig cells of Sirt $1^{F / F}$ mice while immunofluorescence staining of NHERF2 showed a significant increase in the Leydig cells of SF1-Sirt $1^{-1-}$ mice compared to the Leydig cells of Sirt $1^{F / F}$ mice. (M) The relative mRNA levels of Scarb1 were not significantly different in the Leydig cells of Sirt $1^{F / F}$ and SF1-Sirt1 ${ }^{-1-}$ mice upon Sirt1 disruption. (N) Immunoblotting analysis of NHERF2 showed a significant accumulation in autophagy-deficient Leydig cells compared to the Leydig cells of Sirt ${ }^{F / F}$ mice. However, no obvious difference was detected in the levels of NHERF1 and MAP17 in either the Leydig cells of Sirt1 ${ }^{F / F}$ or SF1-Sirt ${ }^{-1-}$ mice. $\beta$-Actin was used as the loading control. (O) Quantification of the signal intensity of NHERF2 shown in $(\mathrm{N})$. Data being represented as mean $\pm \mathrm{SD}$ and ${ }^{* * *} P<0.0001$. (P) Quantification of the signal intensity of MAP17 shown in (N). Data being represented as mean $\pm S D$. (Q) Quantification of the signal intensity of NHERF1 shown in (N). Data being represented as mean $\pm S D$. ( $R$ and $S$ ) Immunofluorescence analyses of MAP17 and NHERF1 showed no obvious differences between the Leydig cells of Sirt $1^{F / F}$ and SF1-Sirt $1^{-1-}$ mice, whereas BODIPY staining showed a sharp decrease in the number of LDs in the Leydig cells of Sirt1-deficient mice compared to Sirt1 ${ }^{F / F}$ mice. (T) Immunofluorescence staining of SR-BI showed a significant decrease whereas NHERF2 showed a significant increase in the Leydig cells of SF1-Sirt1 $1^{-1-}$ mice compared to the Leydig cells of Sirt $1^{F / F}$ mice. (U) Cholesterol (Dil-HDL) absorption was significantly reduced in the Sirt1-deficient Leydig cells compared to the Leydig cells of Sirt $1^{F / F}$ mice. However, Nherf2-knockdown partially rescued the Cholesterol (Dil-HDL) absorption defect in the Leydig cells isolated from SF1-Sirt1 ${ }^{-1-}$ mice.

autolysosomes presence) levels has also been reported in the absence of SIRT1 (Shi et al., 2018). Therefore, we assessed LAMP2 levels by immunofluorescence and found a clear decrease in LAMP2 levels in Sirt1-deficient Leydig cells (Fig. 2L). Collectively, these results suggest a disruptive autophagic process in the steroidogenic cells of SF1-Sirt $1^{-1-}$ mice, and the down-regulation of SR-BI might also come from the disruption of autophagic flux. To find out how SR-BI is regulated in steroidogenic cells via autophagy, relative mRNA levels of Scarb1 was measured in both Sirt ${ }^{F / F}$ and SF1-Sirt $1^{-1-}$ Leydig cells. Surprisingly, Scarb1 mRNA was not decreased but was instead slightly increased in SF1Sirt $1^{-1-}$ Leydig cells (Fig. 2M), suggesting SIRT1 might regulate $\mathrm{SR}-\mathrm{BI}$ via degradation of its negative regulators. MAP17, NHERF1, and NHERF2 are known negative regulators of SR-BI. Therefore, we first measured the levels of these proteins by immunoblotting and found a significant accumulation of NHERF2 in SF1-Sirt $1^{-1-}$ Leydig cells (Fig. 2N and 2O), while the levels of MAP17 and NHERF1 was comparable between Sirt1 $1^{F / F}$ and SF1-Sirt $1^{-1-}$ Leydig cells (Fig. $2 \mathrm{~N}, 2 \mathrm{P}$, and $2 \mathrm{Q}$ ). To verify these findings, we performed immunofluorescence analysis and found no difference between the signals of MAP17 and NHERF1 in Sirt $1^{F / F}$ and SF1-Sirt $1^{-1-}$ mouse testes (Fig. 2R and 2S) but detected a significant increase in NHERF2 in SF1-Sirt1 $1^{-/}$ mouse testes compared with control mouse testes (Fig. 2L). To further investigate the relationship of these proteins, we checked SR-BI and NHERF2 levels in Leydig cells via immunofluorescence. NHERF2 and SR-BI signals were negatively correlated in Sirt $1^{F / F}$ Leydig cells, whereas NHERF2 accumulation led to a considerable reduction in SR-BI level in SF1-Sirt1 ${ }^{-1-}$ Leydig cells (Figs. 2T and S6). Because NHERF2 has previously been reported to be selectively degraded by the autophagy-lysosome pathway (Gao et al., 2018), our new results further suggest that the SIRT1-dependent autophagy-lysosome pathway should be involved in NHERF2 degradation in Leydig cells.

Next, to check whether NHERF2 accumulation is responsible for cholesterol uptake defect observed in SF1-Sirt $1^{-/-}$ Leydig cells, we tested whether Nherf2-knockdown could rescue cholesterol uptake defect in SF1-Sirt $1^{-1-}$ Leydig cells. Therefore, we measured cholesterol uptake in Nherf2knockdown SF1-Sirt $1^{-/-}$and Sirt $1^{\text {FIF }}$ Leydig cells, and found a clear increase in both rates and amounts of cholesterol uptake, as shown by Dil-HDL absorption, compared to Nherf2expressing cells (Fig. 2U). Collectively, NHERF2 seems to act as a connecter between SIRT1 and cholesterol absorption in testicular steroidogenic cells. And, SIRT1 impairment in 


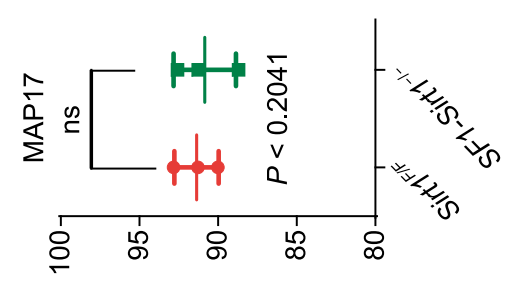

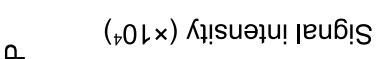
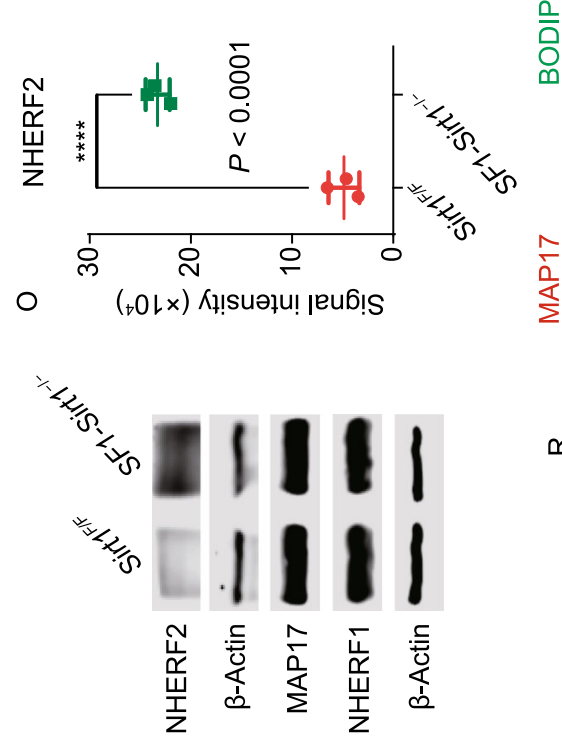

z
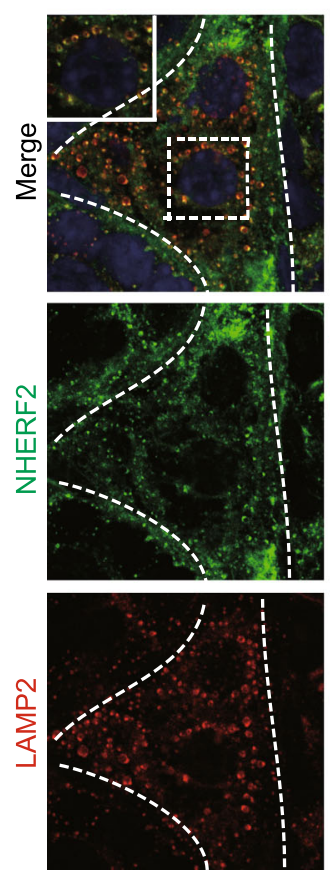

고나!S

$-$

- -เนแ!S-เコS

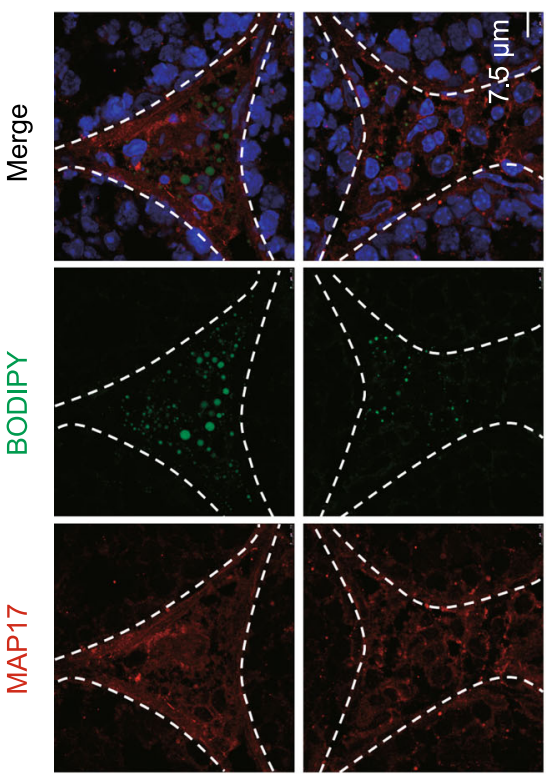

$\propto \quad$ ㅆเㄴ!ㅇ

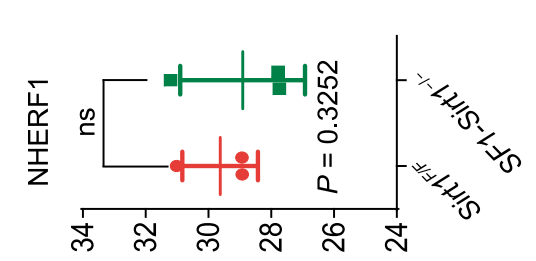

$\left({ }_{t} \mathrm{OLX}\right)$ Kł! suәฺU! jeu6!s
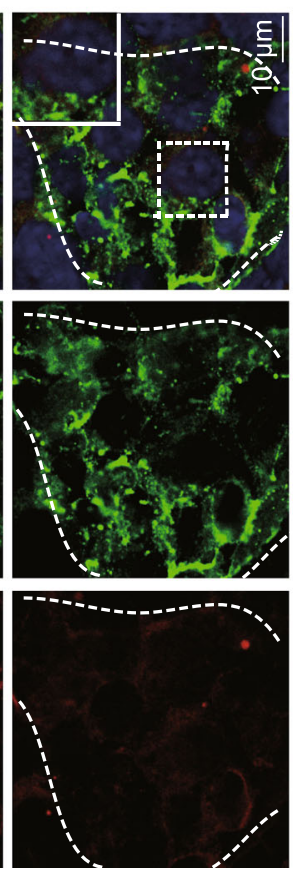

○
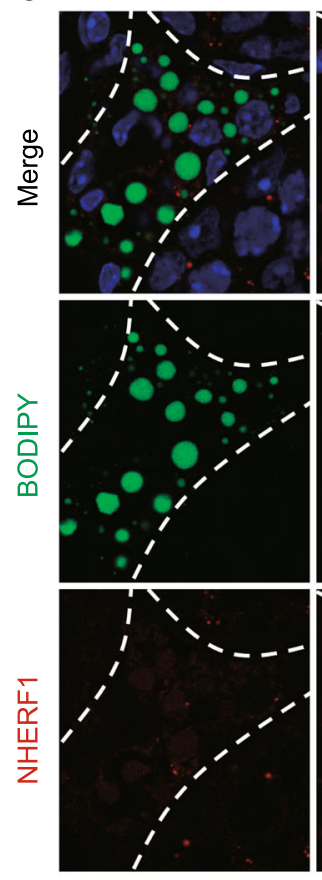

씨닌

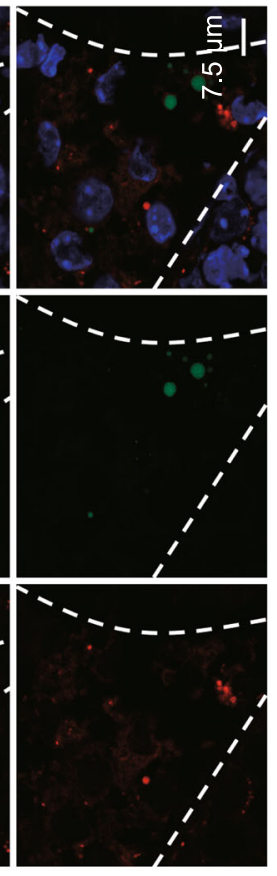

--เLแ!S-เコS

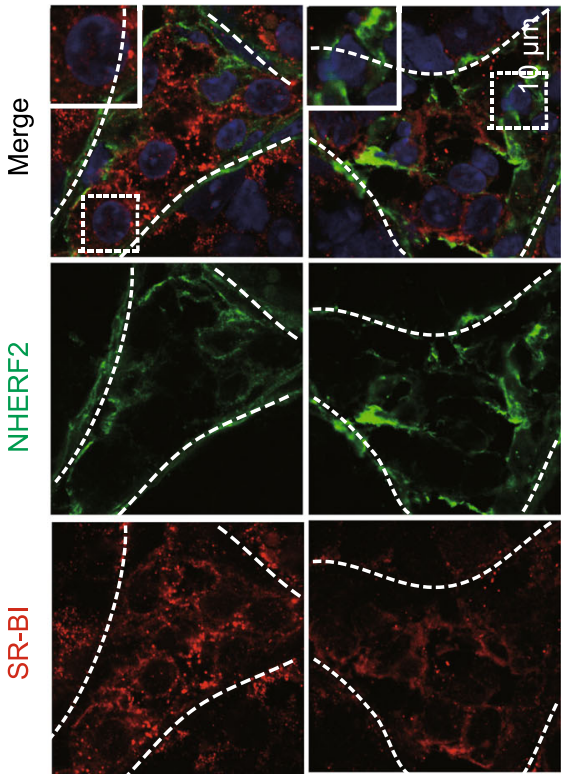

- - L L
/เㄴ!S

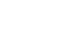

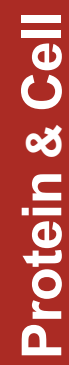

$\omega$

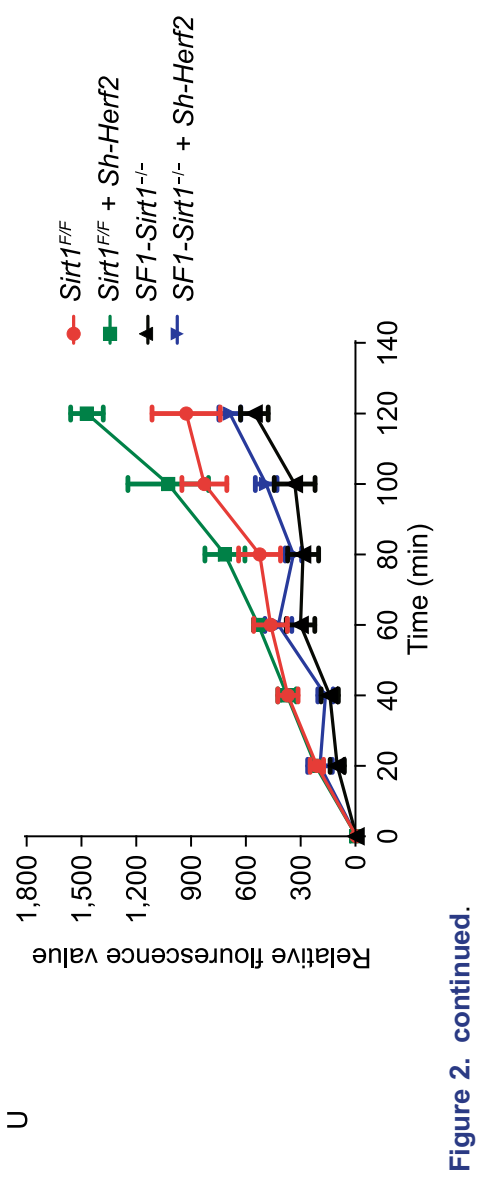


Leydig cells leads to abnormal accumulation of NHERF2 that downregulates $\mathrm{SR}-\mathrm{BI}$, leading to impaired cholesterol uptake and insufficient testosterone biosynthesis. It is noteworthy to mention that decrease in testosterone level was consistent with a significant decrease in steroidogenesis activity and steroidogenesis at the transcriptional and translational level in SF1-Sirt $1^{-1-}$ Leydig cells (Figs. $1 \mathrm{H}-\mathrm{J}$ and S4), and several steroidogenesis factors were also downregulated in Sirt1 conventional knockout mice (Kolthur-Seetharam et al., 2009). As the expression of some steroidogenic enzymes could also be partially rescued in Nherf2-knockdown SF1-Sirt ${ }^{-1-}$ Leydig cells (Fig. S7), the dysfunction of the testosterone synthesis factors might occur in response to the insufficient cholesterol supply. Besides, SIRT1 has been reported to participate in several cellular processes such as gene silencing, glucose and lipid metabolism (Cohen et al., 2004; Chang et al., 2015), SIRT1 may have extensive and multiple functions in the manifold regulation of Leydig cells.

Taken together, SIRT1 deacetylates LC3 in the nucleus, and eventually, LC3 moved from the nucleus to the cytoplasm, where LC3 participates in autophagosome formation by interacting with the other components of the autophagic machinery. NHERF2 is taken up and ultimately degraded via autophagosomes, thus promoting SR-BI expression and accelerating the uptake of cholesterol to fuel the process of steroidogenesis. While in the absence of SIRT1, LC3 fails to deacetylate and translocate to the cytoplasm. Due to autophagic flux disruption, NHERF2 remains intact, thus inhibiting SR-BI expression and cholesterol cannot be efficiently up taken by the Leydig cells, therefore testosterone cannot be synthesized and finally results in LOH (Fig. S8).

\section{FOOTNOTES}

MBK, CL, and FG performed most of the experiments and wrote the manuscript. HG, WWL, TH, and LW performed part of the experiment. GL, HJ, and $W L$ supervised the project and revised the manuscript.

This work was supported by the CAMS Innovation Fund for Medical Sciences (No. 2018-I2M-1-002), the National Natural Science Foundation of China (91649202 and U1904165), and the National Science Fund for Distinguished Young Scholars (81925015).

Muhammad Babar Khawar, Chao Liu, Fengyi Gao, Hui Gao, Wenwen Liu, Tingting Han, Lina Wang, Guoping Li, Hui Jiang, and Wei Li declare that they have no conflict of interest.

The use and care of animals complied with the guideline of the Animal Research Panel of the Committee on Research Practice of the University of Chinese Academy of Sciences, Beijing, P.R. China.

Muhammad Babar Khawar ${ }^{1,3}$, Chao Liu, ${ }^{1,3}$, Fengyi Gao ${ }^{2}$, Hui Gao ${ }^{1}$, Wenwen $\mathrm{Liu}^{1,3}$, Tingting $\mathrm{Han}^{4}$, Lina Wang ${ }^{1,3}$, Guoping Li ${ }^{4 \bowtie}$, Hui Jiang ${ }^{5,6,7,8 \bowtie}$, Wei Li $\mathrm{Li}^{1,3 凶}$ (1)

${ }^{1}$ State Key Laboratory of Stem Cell and Reproductive Biology, Institute of Zoology, Chinese Academy of Sciences, Beijing 100101, China
2 School of Biotechnology and Food, Shangqiu Normal University, Shangqiu 476000, China

${ }^{3}$ University of Chinese Academy of Sciences, Beijing 100049, China

${ }^{4}$ The MOH key Laboratory of Geriatrics, Beijing Hospital, National Center of Gerontology, Beijing 100730, China

${ }^{5}$ Department of Urology, Peking University Third Hospital, Beijing 100191, China

${ }^{6}$ Department of Andrology, Peking University Third Hospital, Beijing 100191, China

7 Department of Reproductive Medicine Center, Peking University Third Hospital, Beijing 100191, China

8 Department of Human Sperm Bank, Peking University Third Hospital, Beijing 100191, China

$\triangle$ Correspondence: guoping_li@yahoo.com (G. Li), jianghui55@163.com (H. Jiang), leways@ioz.ac.cn (W. Li)

Accepted July 13, 2020

\section{OPEN ACCESS}

This article is licensed under a Creative Commons Attribution 4.0 International License, which permits use, sharing, adaptation, distribution and reproduction in any medium or format, as long as you give appropriate credit to the original author(s) and the source, provide a link to the Creative Commons licence, and indicate if changes were made. The images or other third party material in this article are included in the article's Creative Commons licence, unless indicated otherwise in a credit line to the material. If material is not included in the article's Creative Commons licence and your intended use is not permitted by statutory regulation or exceeds the permitted use, you will need to obtain permission directly from the copyright holder. To view a copy of this licence, visit http:// creativecommons.org/licenses/by/4.0/.

\section{REFERENCES}

Bilińska B (1994) Staining with ANS fluorescent dye reveals distribution of mitochondria and lipid droplets in cultured Leydig cells. Folia histochemica et cytobiologica 32:21-24

Chang C, Su H, Zhang D, Wang Y, Shen Q, Liu B, Huang R, Zhou T, Peng C, Wong CC (2015) AMPK-dependent phosphorylation of GAPDH triggers Sirt1 activation and is necessary for autophagy upon glucose starvation. Molecular cell 60:930-940

Chen H, Ge R-S, Zirkin BR (2009) Leydig cells: from stem cells to aging. Molecular and cellular endocrinology 306:9-16

Cohen HY, Miller C, Bitterman KJ, Wall NR, Hekking B, Kessler B, Howitz KT, Gorospe M, de Cabo R, Sinclair DA (2004) Calorie restriction promotes mammalian cell survival by inducing the SIRT1 deacetylase. Science 305:390-392

Danielsen ET, Moeller ME, Yamanaka N, Ou Q, Laursen JM, Soenderholm C, Zhuo R, Phelps B, Tang K, Zeng J (2016) A Drosophila genome-wide screen identifies regulators of steroid hormone production and developmental timing. Developmental cell 37:558-570 
Gao F, Li G, Liu C, Gao H, Wang H, Liu W, Chen M, Shang Y, Wang L, Shi J (2018) Autophagy regulates testosterone synthesis by facilitating cholesterol uptake in Leydig cells. J Cell Biol 217:2103-2119

Hu J, Zhang Z, Shen W-J, Azhar S (2010) Cellular cholesterol delivery, intracellular processing and utilization for biosynthesis of steroid hormones. Nutrition \& metabolism 7:47

Klinefelter GR, Hall PF, Ewing LL (1987) Effect of luteinizing hormone deprivation in situ on steroidogenesis of rat Leydig cells purified by a multistep procedure. Biology of Reproduction 36:769-783

Kolthur-Seetharam U, Teerds K, de Rooij DG, Wendling O, McBurney M, Sassone-Corsi P, Davidson I (2009) The histone deacetylase SIRT1 controls male fertility in mice through regulation of hypothalamic-pituitary gonadotropin signaling. Biology of reproduction 80:384-391

Liu C, Wang H, Shang Y, Liu W, Song Z, Zhao H, Wang L, Jia P, Gao F, Xu Z (2016) Autophagy is required for ectoplasmic specialization assembly in sertoli cells. Autophagy 12:814-832

Liu C, Song Z, Wang L, Yu H, Liu W, Shang Y, Xu Z, Zhao H, Gao F, Wen J (2017) Sirt1 regulates acrosome biogenesis by modulating autophagic flux during spermiogenesis in mice. Development 144:441-451

Midzak AS, Chen H, Papadopoulos V, Zirkin BR (2009) Leydig cell aging and the mechanisms of reduced testosterone synthesis. Molecular and cellular endocrinology 299:23-31

Ouimet M, Barrett TJ, Fisher EA (2019) HDL and Reverse Cholesterol Transport: Basic Mechanisms and Their Roles in Vascular Health and Disease. Circulation research 124:15051518

Shi X, Sun R, Zhao Y, Fu R, Wang R, Zhao H, Wang Z, Tang F, Zhang N, Tian X (2018) Promotion of autophagosome-lysosome fusion via salvianolic acid A-mediated SIRT1 up-regulation ameliorates alcoholic liver disease. RSC advances 8:2041120422

Sinclair M, Grossmann M, Gow PJ, Angus PW (2015) Testosterone in men with advanced liver disease: abnormalities and implications. Journal of gastroenterology and hepatology 30:244-251

Swee DS, Gan E (2019) Late-onset hypogonadism as primary testicular failure. Frontiers in Endocrinology 10:372
Muhammad Babar Khawar, Chao Liu, and Fengyi Gao have contributed equally to this work.

Electronic supplementary material The online version of this article (https://doi.org/10.1007/s13238-020-00771-1) contains supplementary material, which is available to authorized users. 